\title{
Ultra-sonografia do músculo longissimus dorsi de eqüinos da raça Puro-sangue Árabe em treinamento de resistência associado à suplementação prolongada com creatina
}

\section{Ultrassonography images of the longissimus dorsi muscle of Arabian horses in endurance training associated to chronically supplemented with creatine}

\author{
Flora Helena de Freitas D‘Angelis, ${ }^{*}$ Marcílio Dias Silveira da Mota, ${ }^{* *}$ Eduardo Villela Villaça Freitas, ${ }^{*}$, Guilherme de \\ Camargo Ferraz, ${ }^{*}$ André Rodrigues Abrahão, ${ }^{* *}$ José Côrrea de Lacerda-Neto, ${ }^{* * *}$ Antonio de Queiroz-Neto*
}

\begin{abstract}
Resumo
Objetivou-se padronizar medidas ultra-sonográficas do músculo longissimus dorsi de 12 eqüinos da raça Puro-sangue Árabe submetidos a treinamento aeróbico por 90 dias, com alimentação suplementada ou não com creatina monoidratada, a fim de verificar se estas mensurações poderiam auxiliar programas de treinamento com animais desta raça, e se a creatina alteraria as medidas ultra-sonográficas. A suplementação consistiu na administração diária de $75 \mathrm{~g}$ desta substância, misturada à ração durante os 90 dias de treinamento. Para determinar as quantidades diárias da ração comercial fornecida, os animais tiveram o peso corpóreo e o escore corporal avaliados mensalmente. O condicionamento físico foi realizado em esteira rolante de alto desempenho e a intensidade do treinamento foi estipulada mediante o cálculo da $\mathrm{V}_{\mathrm{LAC}}{ }_{4}$. velocidade na qual o lactato sanguíneo atinge $4 \mathrm{mmol} / \mathrm{L}$ ) determinada mensalmente para cada animal. Estabeleceu-se a intensidade do esforço físico individual, em $80 \%$ do limiar aeróbico. Os animais foram avaliados por meio de exames ultra-sonográficos, mensurando-se a área, largura, profundidade de corte transversal e camada de gordura do músculo longissimus dorsi ao final dos oito meses de inatividade e após 30,60 e 90 dias de treinamento. Os resultados da ultra-sonografia revelaram que o treinamento aeróbico associado ou não à suplementação com creatina causou hipertrofia do músculo longissimus dorsi e redução da camada de gordura. O treinamento aeróbico associado à suplementação prolongada com creatina levou ao aumento da medida de profundidade do músculo longissimus dorsi.
\end{abstract}

Palavras-chave: longissimus dorsi; ultra-sonografia; treinamento aeróbico; eqüino.

\begin{abstract}
The goal of this study was to standardize ultrassonographic measurements of the longissimus dorsi muscle in twelve Arabian horses submitted to an aerobic training for 90 days, with feed supplemented or not with monohydrated creatine, in order to find out if these measurements could help training programs of this breed of horses. Another point was to find out if creatine supplementation does enhance ultrassonographic measurements. The creatine supplementation was carried out by daily administration of $75 \mathrm{~g}$ of this substance, for 90 training days. To determine the daily administration of the commercial ration supplied, the animals had the corporeal height and the corporeal score monthly considered. The conditioning physical program was conducted in a treadmill and the training protocol was adjusted after $\mathrm{V}_{\mathrm{LAC}_{4}}$ (velocity in which the lactato blood reachs $4 \mathrm{mmol} /$ L) determination which was set monthly for each horse. The intensity of effort was determined as $80 \%$ of the aerobic threshold of each horse. The animals were ultrassonographically examined measurement of the area, width of transversal cut, depth of transversal cut and fat layer of the longissimus dorsi muscle on each groups at end of eight months of inactivity and after 30,60 and 90 training days. The ultrassonographical results showed that, the aerobic training associated or not to creatine supplementation caused longissimus dorsi hypertrophy and reduction in the thickness of the fat layer. The aerobic training associated to chronically supplemented with creatine made the rising of the profundity measure of longissimus dorsi muscle.
\end{abstract}

Keywords: Longissimus dorsi, ultrassonography, endurance, horse.

\section{Introdução}

Várias substâncias ergogênicas têm sido empregadas na melhoria do potencial atlético eqüino. Uma dela é a creatina, uma substância amplamente utilizada como nutracêutico em diversos esportes eqüestres. A creatina é um composto orgânico encontrado em abundância nos músculos esqueléticos e desempenha um papel central na disponibilidade de energia do músculo (Greenhaff, 1997). Quando esta é fosforilada

\footnotetext{
* Departamento de Morfologia e Fisiologia Animal,Universidade Estadual Paulista (UNESP) - Faculdade de Ciências Agrárias e Veterinárias Campus Jaboticabal; via de acesso Prof. Paulo Donato Castellane, s/n Jaboticabal SP, Brasil - CEP 14884-900 - telefone: (16) 3209 2654 e-mail aqueiroz@fcav.unesp.br

** Departamento de Melhoramento e Nutrição Animal, UNESP - Faculdade de Medicina Veterinária e Zootecnia - Campus de Botucatu, Distrito de Rubião Junior, Botucatu, São Paulo, Brasil - CEP 18618-000 - Caixa Postal: 502, FAX: (14) 6821-3902 - telefone: (14) 6802-6179

***Departamento de Clínica e Cirurgia, UNESP, campus Jaboticabal.
} 
pela enzima creatina cinase em creatina fosfato, doa o ortofosfato inorgânico necessário para refosforilar ADP em ATP, liberando energia e mantendo, desta forma, a contração muscular (Brannon et al., 1996). Parece claro, atualmente, que a suplementação, com altas doses desta substância, por tempo curto (1 semana), acarreta uma capacidade maior de realizar exercícios máximos intermitentes (Casey et al., 1996; Vandenberghe et al., 1996; Volek et al., 1997). Com relação aos possíveis benefícios decorrentes da ingestão por tempo prolongado (mais que uma semana) de $\mathrm{Cr}$, parece que embora não existam, ainda, evidências científicas que justifiquem seu uso, este já se encontra largamente disseminado como uma prática ergogênica padrão entre várias categorias de atletas, inclusive entre cavalos.

Recentemente, foi demonstrado que a suplementação com creatina por tempo prolongado estimula a hipertrofia muscular durante treinamento de alta resistência, em atletas humanos (Vandenberghe et al., 1997). Sabe-se, também, que a creatina aumentou o peso corporal em estudo realizado em atletas humanos (Balson et al., 1995). Estas afirmações justificam a necessidade de pesquisas relacionadas com avaliação da composição corporal e a suplementação com creatina na dieta de eqüinos atletas.

Diversas regiões do corpo do animal podem ser mensuradas na avaliação da composição corporal. O músculo longissimus dorsi foi escolhido para as mensurações ultra-sonográficas desse experimento, por ser apontado como um dos mais utilizados durante o exercício e o treinamento e o que melhor avalia o desempenho do cavalo em corridas. Afirmação bem avaliada pelos dados obtidos por Dobec et al. (1994), que demonstraram uma relação significativa entre o tamanho da área de corte transversal do músculo longissimus dorsi e o número de vitórias obtidas pelos animais em competições de corridas.

Finalmente, as aparentes contradições e dúvidas existentes sobre a utilização da creatina como complemento alimentar indicam claramente a necessidade de estudos adicionais com essa substância, antes de poder prescrevê-la como complemento alimentar de cavalos atletas.

Poucos trabalhos foram realizados com objetivo de padronizar medidas ultra-sônicas tomadas no músculo longissimus dorsi e o desempenho de eqüinos atletas em diferentes modalidades esportivas, devido a este fato, objetivou-se conhecer o comportamento existente entre medidas ultrasonográficas da área de corte transversal, profundidade, largura e espessura da camada de gordura realizadas no músculo longissimus dorsi relacionadas com o treinamento de resistência de cavalos Puro-sangue Árabe, sujeitos a suplementação ou não com creatina.

\section{Material e métodos}

\section{Animais e manejo}

Foram utilizados 12 eqüinos, machos e fêmeas, Puro-sangue Árabe (PSA), com peso corpóreo médio inicial de 389, $23 \pm$ $25,17 \mathrm{Kg}$ (média \pm desvio-padrão) e idade média de $8,60 \pm 3,30$ anos, provenientes do Setor de Eqüideocultura da FCAV-UNESP, Jaboticabal. Os animais selecionados aleatoriamente para comporem os grupos experimentais foram avaliados através de exames clínicos e laboratoriais completos, sem sinais ou sintomas compatíveis com lesões musculoesqueléticas, casqueados e desverminados quando necessário. Os animais foram distribuídos em grupos de acordo com o Quadro 1.
Quadro 1- Distribuição dos animais de acordo com os grupos experimentais

\begin{tabular}{|c|c|c|c|}
\hline $\begin{array}{c}\text { Duração do } \\
\text { treinamento } \\
\text { (dias) }\end{array}$ & Suplementação & $\begin{array}{c}\text { Grupo } \\
\text { experimental }\end{array}$ & $\begin{array}{c}\text { Número de } \\
\text { animais }\end{array}$ \\
\hline 0 & Não & $\mathrm{D}$ & 12 \\
\hline 30 & $\mathrm{Sim}$ & $\mathrm{Tcr}_{30}$ & 6 \\
\cline { 2 - 4 } & $\mathrm{Não}$ & $\mathrm{T}_{30}$ & 6 \\
\hline 60 & $\mathrm{Sim}$ & $\mathrm{Tcr}_{60}$ & 6 \\
\cline { 2 - 4 } & $\mathrm{Não}$ & $\mathrm{T}_{60}$ & 6 \\
\hline \multirow{2}{*}{90} & Sim & $\mathrm{Tcr}_{90}$ & 6 \\
\cline { 2 - 4 } & não & $\mathrm{T}_{90}$ & 6 \\
\hline
\end{tabular}

$\mathrm{D}$ : animais antes do treinamento e da suplementação com creatina; $T_{30}$, $\mathrm{T}_{60}, \mathrm{~T}_{90}$ : animais treinados por 30,60 e 90 dias, respectivamente, e sem suplementação com creatina; $\mathrm{Tcr}_{30}, \mathrm{Tcr}_{60}, \mathrm{Tcr}_{90}$ : animais treinados $\mathrm{e}$ suplementados com creatina por 30,60 e 90 dias, respectivamente.

Os 12 animais que constituíram o grupo $\mathrm{D}$ (grupo destreinado) permaneceram por um período de oito meses de inatividade antes de iniciar o treinamento, em piquetes com água e pastagem de Brachiaria sp. ad libitum, recebendo suplementação com feno de Cynodon sp. e sal mineralizado. Os animais dos grupos $T_{30}, T_{60}, T_{90}, T_{c r}, T_{c r}, T_{60} T_{90}$ foram submetidos ao mesmo programa de treinamento e arraçoamento, sendo que, para os grupos $\mathrm{Tcr}_{30}, \mathrm{Tcr}_{60}, \mathrm{Tcr}_{90}$ foi fornecida, diariamente, misturada a ração comercial, ${ }^{1} 75 \mathrm{~g}$ de creatina monoidratada. ${ }^{2}$ Para determinar as quantidades diárias da ração comercial, os animais tiveram o peso e o escore corpóreo avaliados mensalmente utilizando o protocolo preconizado por Henneke et al. (1983).

\section{Protocolo de treinamento}

Objetivando-se viabilizar a simulação de uma prova de enduro eqüestre com $60 \mathrm{~km}$ de extensão, os animais foram submetidos a um programa de treinamento aeróbico. O condicionamento físico de cada animal foi realizado em sala climatizada, na qual instalou-se uma esteira rolante de alto desempenho. ${ }^{3}$ O programa de treinamento foi realizado exclusivamente em esteira rolante e a velocidade (intensidade) do mesmo foi definida como sendo $80 \%$ da $\mathrm{V}_{\text {lac } 4}$ (velocidade na qual a concentração de lactato sangüíneo atinge $4 \mathrm{mmol} / \mathrm{L}$ ). Para a determinação da $V_{\text {lac4 }}$ os animais eram submetidos a um teste ergométrico em que a velocidade era aumentada (2, 4, 6 e $8 \mathrm{~m} /$ s) a cada minuto com a esteira inclinada a $6 \%$. Desta forma, determinou-se através de análise de regressão, a velocidade na qual as concentrações de lactato sangüíneo alcançava o limiar aeróbico $(4 \mathrm{mmol} / \mathrm{L})$. Ao final de cada período de treinamento (30 dias), realizava-se um novo teste ergométrico para estabelecimento de uma nova $V_{\text {LAC4. }}$ Assim, mensalmente determinava-se a velocidade de treinamento para cada animal.

A freqüência do treinamento compreendeu a realização de exercícios três vezes por semana (dias alternados). No primeiro mês de treinamento, cada animal percorreu em média $10 \mathrm{~km}$ em 50 minutos. No segundo mês, a distância percorri-

\footnotetext{
${ }^{1}$ Nutriage MIX Guabiå, Campinas, SP, Brasil.

2 Vetnil, Louveira, SP, Brasil.

3 Esteira Galloper $5500^{\circledR}$ SAHINCO Ltda, Palmital, SP
} 
da em cada exercício foi aumentada para $15 \mathrm{~km}$ com duração média de 60 minutos. No último mês, todos os cavalos percorreram $20 \mathrm{~km}$ por sessão, com duração média de 90 minutos. Adicionalmente, neste mês foi instituído, uma vez por semana, treinamento do tipo jogo de velocidade, ou seja fartlek. Nesse exercício foi empregado um protocolo que iniciava com um período de aquecimento a $4,0 \mathrm{~m} / \mathrm{s}$ durante 10 minutos. $\mathrm{Na}$ etapa seguinte, a esteira era inclinada para $5 \%$ sendo a velocidade aumentada para $8 \mathrm{~m} / \mathrm{s}$ com duração de 10 minutos. Na próxima etapa, a velocidade da esteira e a duração do exercício eram reduzidas para $2,5 \mathrm{~m} / \mathrm{s}$ e 5 minutos respectivamente, sem inclinação. A seguir, a esteira era inclinada a 5\% e a velocidade aumentada para $10 \mathrm{~m} / \mathrm{s}$ por 5 minutos. Novamente, reduzia-se a velocidade para $2,5 \mathrm{~m} / \mathrm{s}$ por 5 minutos se inclinação. A velocidade da esteira era novamente aumentada para $5,0 \mathrm{~m} / \mathrm{s}$ com inclinação de $10 \%$ e duração de 20 minutos. Logo em seguida, aumentava-se a velocidade para $12 \mathrm{~m} /$ $\mathrm{s}$, sem inclinação, com duração de 3 minutos. Os animais finalizavam a atividade física com um período de desaquecimento a 2,0m/s por 10 minutos sem inclinação.

\section{Avaliação ultra-sonográfica}

Com o intuito de avaliar o efeito do treinamento e da suplementação com creatina sobre a musculatura esquelética foram realizadas, por meio de imagem ultra-sonográfica, mensurações da área de corte transversal $(\mathrm{mm})$, largura de corte transversal $(\mathrm{mm})$, profundidade de corte transversal $(\mathrm{mm})$ e camada de gordura $(\mathrm{mm})$ do músculo longissimus dorsi em todos os grupos, ao final dos oito meses de inatividade (grupo D) e após 30, 60 e 90 dias de treinamento. Para tanto, foi utilizado aparelho de ultra-som ${ }^{4}$ equipado com um programa de computador específico ${ }^{5}$ para a avaliação muscular. Este tipo de aparelho é especializado na versão "Bmode" e tem habilidade de produzir imagens quase que instantaneamente com objetos em movimento.

As mensurações foram realizadas na altura das duas últimas costelas esquerda conforme Mota (2002), ${ }^{6}$ com o animal em posição aprumada e o músculo longissimus dorsi relaxado.

\section{Análise estatística}

O delineamento experimental adotado foi de parcela subdividida, sendo que os tratamentos foram parcelas e os três meses de treinamento as subparcelas. O peso corpóreo e as variáveis ultra-sonográficas foram avaliados mediante análise de variância (Sampaio, 1998), utilizando-se para a comparação entre as médias o teste de Tukey $(P<0,05)$. Para análise do escore corporal foi utilizado o teste não-paramétrico de Friedman.

\section{Resultados}

Analisando os dados dispostos na Tabela 1, observa-se que os valores médios de peso corpóreo não diferiram significativamente $(P>0,05)$ entre os grupos ao longo do treinamento. Da mesma forma, os valores de escore corporal não apresentaram diferença significativa entre os grupos e tempos de treinamento, analisando os dados através do emprego da análise estatística não-paramétrica.

\footnotetext{
4 Pie Medical-scanner 200, modelo 51B04UM02 3,5/5 Mhz DF TRD Linear, EUA.

5 Software Eview - Echo Image Viewer, Copyright Pie Medical, 1996, EUA.

${ }^{6}$ Comunicação pessoal
}

A Tabela 2 mostra as médias e o desvio-padrão de mensurações das variáveis área de corte transversal $\left(\mathrm{cm}^{2}\right)$, profundidade $(\mathrm{mm})$, largura $(\mathrm{mm})$ e espessura da camada de gordura $(\mathrm{mm})$ obtidas no exame ultra-sonográfico do músculo longissimus dorsi (Figura 1).

$\mathrm{Na}$ análise dos resultados da área de corte transversal $\left(\mathrm{cm}^{2}\right)$, verificou-se um aumento significativo dessa variável $(P<0,05)$ ao comparar o grupo $\mathrm{D}$ com os grupos $\mathrm{T}_{30}, \mathrm{~T}_{60}$ e $\mathrm{T}_{90}$. Na comparação das médias dos animais do grupo $D$ com os animais do grupo $\mathrm{Tcr}_{30}, \mathrm{Tcr}_{60}$ e $\mathrm{Tcr}_{90}$, nota-se que também houve aumento estatisticamente significativo $(P<0,05)$ da área de corte transversal. Comparando-se os dados dos grupos $\mathrm{T}_{30}, \mathrm{~T}_{60}, \mathrm{~T}_{90}$ com os dos grupos $\mathrm{Tcr}_{30}, \mathrm{Tcr}_{60}$ e $\mathrm{Tcr}_{90}$, respectivamente, observou-se que os números médios dos mesmos não diferiram significativamente $(P>0,05)$ entre si quanto à área de corte transversal.

Quanto à variável profundidade de corte transversal $(\mathrm{mm})$ do músculo longissimus dorsi, não se obteve diferença estatisticamente significativa $(P>0,05)$ na comparação das médias entre o grupo $\mathrm{D}$ e os grupos $\mathrm{T}_{30}, \mathrm{~T}_{60}$ e $\mathrm{T}_{90}$ Porém, constatou-se diferença estatisticamente significativa $(P<0,05)$ entre os grupos $\operatorname{Tcr}_{30}, \operatorname{Tcr}_{60}$ e $\operatorname{Tcr}_{90}$ e o grupo D. Não foi evidenciada diferença significativa $(P>0,05)$ comparando as médias dos grupos suplementados com creatina com os grupos $\mathrm{T}_{30}, \mathrm{~T}_{60}$ e $\mathrm{T}_{90}$.

$\mathrm{Na}$ análise da largura de corte transversal $(\mathrm{mm})$ do referido músculo, observa-se que os valores médios dos grupos $\mathrm{T}_{30}$, $\mathrm{T}_{60}, \mathrm{~T}_{90}, \mathrm{Tcr}_{30}, \mathrm{Tcr}_{60}$ e $\mathrm{Tcr}_{90}$ comparando-se ao grupo D não foram significativamente $(P>0,05)$ diferentes.

Para a variável espessura da camada de gordura $(\mathrm{mm})$ demonstrou-se que não houve diferença estatisticamente significativa na comparação entre as médias do grupo $D$ com o grupo $\mathrm{T}_{30}$, da mesma forma, com o grupo $\mathrm{Tcr}_{30}$. No entanto, analisando 0 grupo $\mathrm{D}$ com as médias obtidas em $\mathrm{T}_{60}, \mathrm{Tcr}_{60}$, $\mathrm{T}_{90}$ e $\mathrm{Tcr}_{90}$, denotou-se diminuição significativa $(\mathrm{P}<0,05)$ da espessura da camada de gordura nesses grupos. Não foi observada diferença significativa na comparação entre os grupos $\mathrm{T}_{30}$ e $\mathrm{Tcr}_{30}, \mathrm{~T}_{60}$ e $\mathrm{Tcr}_{60}$ e $\mathrm{T}_{90}$ com $\mathrm{Tcr}_{90}$.

\section{Discussão e conclusão}

A baixa quantidade de gordura corporal e o aumento da área ocupada pelo músculo esquelético são de grande valor para estimar o potencial atlético de eqüinos competidores de elite, tanto em eventos de longa distância como em corridas de curta distância (Kearns et al., 2002a e b)

Nosso trabalho revelou um aumento de tamanho da área de corte transversal do músculo longissimus dorsi em todos os grupos experimentais ao longo do treinamento. Constata-se que o treinamento induziu esse aumento, caracterizando uma melhoria na composição corporal dos animais, por aumentar a massa livre de gordura.

O treinamento mas não a creatina influenciou no aumento da área de corte transversal e diminuição da camada de gordura. Por outro lado, a suplementação com creatina associada com o treinamento aeróbico causaram aumento na profundidade muscular, indicando ser essa substância responsável pela hipertrofia muscular, visualizada na mensuração desta variável.

Balsom et al. (1993) e Stroud et al. (1994) concordam que a Cr não produz efeitos sobre exercícios de baixa intensidade (enduro), em pesquisas com atletas humanos. Por outro lado, Low et al. (1996) afirmam que a suplementação com creatina 
Tabela 1 - Peso corpóreo e escore corporal de 12 eqüinos da raça Puro-sanaeróbico por 30, 60 e 90 dias, com e sem suplementação com creatina monoidratada (UNESP - Jaboticabal, SP).

Tabela 2 - Variáveis morfométricas analisadas no músculo longissimus dorsi, obtidas através de exame ultrasonográficco de 12 eqüinos da raça Purosangue Árabe, submetidos a treinamento aeróbico por 30, 60 e 90 dias, com e sem suplementação com creatina monoidratada (UNESP-Jaboticabal, $\mathrm{SP})$. gue Árabe, submetidos a treinamento

\begin{tabular}{|c|c|c|c|c|c|c|c|}
\hline \multirow{3}{*}{$\begin{array}{l}\text { Variáveis } \\
\text { fisiológicas }\end{array}$} & \multicolumn{7}{|c|}{ TRATAMENTOS } \\
\hline & \multirow{2}{*}{$\begin{array}{c}\begin{array}{c}\text { Antes do } \\
\text { treinamento }\end{array} \\
\mathrm{D} \\
(\mathrm{n}=12)\end{array}$} & \multicolumn{2}{|c|}{$\begin{array}{c}30 \text { dias de } \\
\text { treinamento }\end{array}$} & \multicolumn{2}{|c|}{$\begin{array}{l}60 \text { dias de } \\
\text { treinamento }\end{array}$} & \multicolumn{2}{|c|}{$\begin{array}{l}90 \text { dias de } \\
\text { treinamento }\end{array}$} \\
\hline & & $\begin{array}{c}\mathrm{T}_{30} \\
(\mathrm{n}=6)\end{array}$ & $\begin{array}{l}\mathrm{Tcr}_{30} \\
(\mathrm{n}=6) \\
\end{array}$ & $\begin{array}{c}\mathrm{T}_{60} \\
(\mathrm{n}=6)\end{array}$ & $\begin{array}{l}\operatorname{Tcr}_{60} \\
(\mathrm{n}=6) \\
\end{array}$ & $\begin{array}{c}\mathrm{T}_{90} \\
(\mathrm{n}=6)\end{array}$ & $\begin{array}{l}\operatorname{Tcr}_{90} \\
(n=6) \\
\end{array}$ \\
\hline o cornóreo & $391,00^{A}$ & $390,67^{A}$ & $404,50^{A}$ & $382,80^{\mathrm{A}}$ & $388,67^{A}$ & $387,50^{A}$ & $398,00^{A}$ \\
\hline$(\mathrm{Kg})^{*}$ & $\begin{array}{c} \pm \\
25.43\end{array}$ & $\stackrel{ \pm}{ \pm}$ & $\begin{array}{c} \pm \\
19.57\end{array}$ & $\stackrel{ \pm}{ \pm}$ & $\begin{array}{c} \pm \\
15.87\end{array}$ & $\begin{array}{c} \pm \\
29.01\end{array}$ & $\begin{array}{c} \pm \\
27.64\end{array}$ \\
\hline Escore corporal** & $5^{\mathrm{NS}}$ & $5^{\mathrm{NS}}$ & $5^{\mathrm{NS}}$ & $5^{\mathrm{NS}}$ & $5^{\mathrm{NS}}$ & $5^{\mathrm{NS}}$ & $5^{\mathrm{NS}}$ \\
\hline
\end{tabular}

D: animais antes do treinamento e da suplementação com creatina; $T_{30}, T_{60}, T_{90}$ : animais treinados por 30, 60 e 90 dias, respectivamente, e sem suplementação com creatina; $\operatorname{Tcr}_{30}, \mathrm{Tcr}_{60}, \mathrm{Tcr}_{90}$ : animais treinados e suplementados com creatina por 30,60 e 90 dias, respectivamente.

* Média \pm desvio-padrão. Médias na mesma linha seguidas de letras diferentes diferem significativamente $(p<0,05)$ pelo teste de Tukey.

**De acordo com Henneke et al. (1983). NS: diferença não significativa (teste não-paramétrico de Friedman)

\begin{tabular}{|c|c|c|c|c|c|c|c|}
\hline \multirow{3}{*}{$\begin{array}{l}\text { Variáveis ultra- } \\
\text { sonográficas }\end{array}$} & \multicolumn{7}{|c|}{ TRATAMENTOS } \\
\hline & \multirow{2}{*}{$\begin{array}{c}\begin{array}{c}\text { Antes do } \\
\text { treinamento }\end{array} \\
D \\
(n=12)\end{array}$} & \multicolumn{2}{|c|}{$\begin{array}{l}30 \text { dias de } \\
\text { treinamento }\end{array}$} & \multicolumn{2}{|c|}{$\begin{array}{c}60 \text { dias de } \\
\text { treinamento }\end{array}$} & \multicolumn{2}{|c|}{$\begin{array}{c}90 \text { dias de } \\
\text { treinamento }\end{array}$} \\
\hline & & $\begin{array}{c}\mathrm{T}_{30} \\
(\mathrm{n}=6)\end{array}$ & $\begin{array}{l}\mathrm{Tcr}_{30} \\
(\mathrm{n}=6)\end{array}$ & $\begin{array}{c}\mathrm{T}_{60} \\
(\mathrm{n}=6)\end{array}$ & $\begin{array}{l}\mathrm{Tcr}_{60} \\
(\mathrm{n}=6)\end{array}$ & $\begin{array}{c}\mathrm{T}_{90} \\
(\mathrm{n}=6)\end{array}$ & $\begin{array}{l}\operatorname{Tcr}_{90} \\
(n=6)\end{array}$ \\
\hline & $73,25^{A}$ & $83,58^{\mathrm{B}}$ & $88,70^{B}$ & $83,71^{\mathrm{B}}$ & $88,81^{B}$ & $85,35^{B}$ & $89,79^{B}$ \\
\hline $\begin{array}{l}\text { Area de corte } \\
\text { transversal }\left(\mathrm{cm}^{2}\right)\end{array}$ & \pm & \pm & \pm & $2 \frac{ \pm}{94}$ & $2 \frac{ \pm}{31}$ & \pm & \pm \\
\hline Profundidade de & $74,86^{A}$ & $81.19^{\mathrm{AB}}$ & $82,84^{\mathrm{B}}$ & $80,57^{A B}$ & $82,91^{\mathrm{B}}$ & $78,77^{\mathrm{AB}}$ & $83,05^{\mathrm{B}}$ \\
\hline $\begin{array}{l}\text { corte transversal } \\
(\mathrm{mm})\end{array}$ & 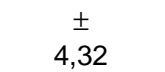 & $\begin{array}{c} \pm \\
4,14\end{array}$ & $\begin{array}{c} \pm \\
3,73\end{array}$ & $\begin{array}{c} \pm \\
4,05\end{array}$ & $\begin{array}{c} \pm \\
2,97\end{array}$ & $\begin{array}{c} \pm \\
3,28\end{array}$ & $\begin{array}{c} \pm \\
1,97\end{array}$ \\
\hline Largura de corte & $160,76^{A}$ & $169,37^{\mathrm{A}}$ & $169,22^{A}$ & $168,51^{\mathrm{A}}$ & $170,28^{A}$ & $169,57^{A}$ & $168,70^{A}$ \\
\hline & $\stackrel{ \pm}{6.13}$ & $\stackrel{ \pm}{4.05}$ & $\begin{array}{c} \pm \\
5.98\end{array}$ & $\begin{array}{c} \pm \\
5.97\end{array}$ & $\stackrel{ \pm}{2.87}$ & $\stackrel{ \pm}{ \pm .90}$ & $\begin{array}{c} \pm \\
4.29\end{array}$ \\
\hline & $4,58^{\mathrm{A}}$ & $3,03^{A}$ & $3,27^{A}$ & $2,47^{\mathrm{B}}$ & $2,79^{\mathrm{B}}$ & $2,69^{\mathrm{B}}$ & $2,47^{\mathrm{B}}$ \\
\hline & $\begin{array}{c} \pm \\
0,87\end{array}$ & $\begin{array}{c} \pm \\
0,74\end{array}$ & $\begin{array}{c} \pm \\
0,74\end{array}$ & $\begin{array}{c} \pm \\
0,90\end{array}$ & $\begin{array}{c} \pm \\
0,85\end{array}$ & $\begin{array}{c} \pm \\
0,26\end{array}$ & $\begin{array}{c} \pm \\
0,21\end{array}$ \\
\hline
\end{tabular}

$\mathrm{D}$ : animais antes do treinamento e da suplementação com creatina; $T_{30}, T_{60}, T_{90}$ : animais treinados por 30, 60 e 90 dias, respectivamente, e sem suplementação com creatina; $\mathrm{Tcr}_{30}, \mathrm{Tcr}_{60}, \mathrm{Tcr}_{90}$ : animais treinados e suplementados com creatina por 30,60 e 90 dias, respectivamente.

Média \pm desvio-padrão. Médias na mesma linha seguidas de letras diferentes diferem significativamente $(p<0,05)$ pelo teste de Tukey.

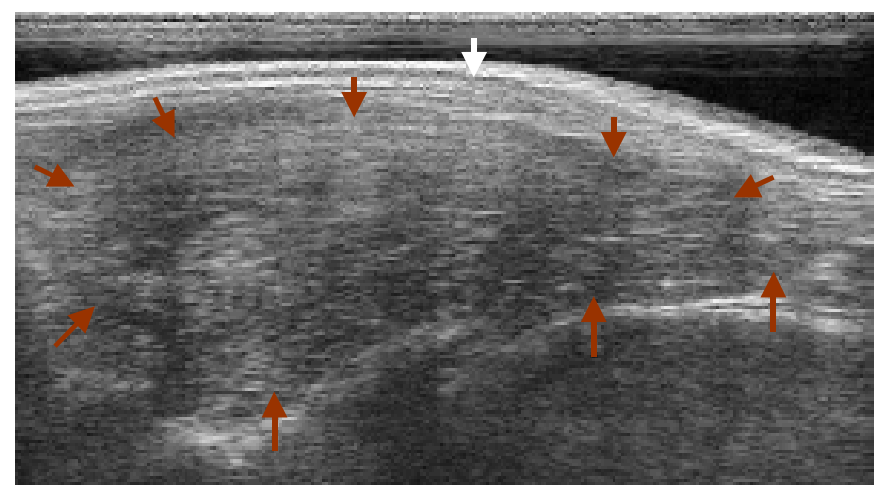

pode aumentar o volume celular causado pela elevação da osmolaridade mioplásmica. Uma teoria bem aceita atualmente é que a creatina causa retenção de água, aumentando o tamanho da célula por modificação no gradiente de concentração (Haussinger et al., 1993). Isto pode explicar o aumento de peso visualizado em humanos após a ingestão de creatina na dieta. Embora não tenha sido averiguado um aumento de peso significativo nos animais deste experimento, ao longo do período de suplementação com a creatina, sabe-se que o eqüino tem que apresentar um ganho de peso considerável para demonstrar um aumento estatisticamente significativo (Schuback et al., 2000).

Os resultados desse experimento demonstram que os animais mantiveram um escore corporal moderado e sem alteração de peso corpóreo ao longo do treinamento e da suplementação com creatina, evidenciando equilíbrio entre a ingestão de alimentos e gasto calórico durante todo o experimento.

Fúsa 1 - Figura ilustrativa visualizando a imagem ultra-sonográfica do Setas vermelhas delimitam a área mensurada do músculo, e seta branca demonstra a imagem da camada de gordura
A avaliação ultra-sonográfica da camada de gordura do músculo longissimus dorsi geralmente é utilizada para a determinação da qualidade de carcaça de animais destinados ao consumo de carne. No entanto, esse trabalho propôs o emprego desta técnica para a avaliação do treinamento, tentando estabelecer uma correlação entre o tamanho da área de 
corte transversal e a espessura da camada de gordura em uma porção anatômica estabelecida, com um programa de treinamento aeróbico preestabelecido. A área de corte transversal aumentou e a espessura da camada de gordura sofreu redução após o período de treinamento, sendo que o peso e o adequado escore corporal apresentados pelos animais não sofreram modificações. Dessa forma, durante as avaliações mensais, notou-se a diminuição de tecido adiposo e aumento da massa muscular devido à intensidade do exercício aeróbico utilizado.

Este efeito observado é sustentado por Sparling e Cureton (1983) e Kearns et al. (2000a, b) que afirmam haver uma correlação negativa entre a quantidade de gordura corporal e o desempenho atlético de cavalos em corridas e em eventos de longa distância. Lawrence et al. (1992) estudaram a influencia da massa de gordura em cavalos de enduro, usando Árabes e Cruza-árabes. A percentagem de gordura corporal nos cavalos competindo em um percurso de $241,35 \mathrm{~km}$ foi em torno de 7,8\%. Quando se comparou o desempenho durante a competição e a percentagem obtida dessa variável, os cavalos que obtiveram maior sucesso no percurso possuíam menor gordura corporal $(6,5 \%)$ em relação aos animais que não finalizaram a prova (11\%).

Embora a composição corporal seja considerada um fator importante na avaliação da saúde animal, poucos dados foram publicados referentes à avaliação da composição corporal em eqüinos e em cavalos atletas durante as competições esportivas (Kearns et al., 2002a e b). Alguns desses procedimentos são caros em termos de pessoal e equipamento, e

\section{Referências}

BALSOM, P. D.; HARRIDGE, S. D. R.; SÖDERLUND, K.; SJÖDIN, B.; EKBLOM, B. Creatine supplementation per se does not enhance endurance exercise performance. Acta Physiol Scand, v. 149, p. 521523, 1993.

BALSON, P.D.; SöDERLUND, K.; SJöDIN, B.; EKBLOM, B. Skeletal muscle metabolism during short duration high-intensity exercise influence of creatine supplementation. Acta Physiol Scand, v. 154, p. 303-310, 1995.

BRANNON, T. A.; ADAMS, G. R.; CONNIFF, C. L.; BALDWIN, K. M. Effects of creatine loading and training on running performance and biochemical properties of rat skeletal muscle. Med Sci Sport Exerc, v. 29, p. 489-495, 1996.

CASEY, A.; CONSTANTIN-TEODOSIU, D.; HOWELL, S.; HULTMAN, E.; GREENHAFF, P. L. Creatine ingestion favorably affects performance and muscle metabolism during maximal exercise in humans. Am J Physiol, v. 271, p. E31-37, 1996.

DOBEC, R. L;, BORGER, M. L.; NEAL, S. M. Correlation of real-time ultrasonic measure of fat and longisimus muscle area in standardbred horses with lifetime racing records and lifetime winnings. J Anim Sci, v. 72, p. $45,1994$.

GREENHAFF, P. L. Review. The nutritional biochemistry of creatine. Nutr Biochemistry, v. 8, p. 610-618, 1997.

HÄUSSINGER, D.; ROTH, E.; LANG, F.; GEOK, W. Cellular hydration state: an important determinant of protein catabolism in health and disease. Lancet, v. 341, p. 1330-1332, 1993.

HENNEKE, D. R.; POTTER, G. D.; KRIEDER, J. L. Relationship between condition score, physical measurements and body fat percentage in mares. Equine Vet J, v. 15, p. 371-372, 1983.

KEARNS, C. F.; McKEEVER, K. H.; ABE, T. Review. Overview of horse body composition and muscle architecture: Implications for performance. Vet Rec, v. 164, p. 224-234, 2002a.

. Fat-free mass is related to one mile race performance in elite Standardbred horses. Vet J, v. 163, p. 1-7, 2002b. não são utilizados por serem de difícil manuseio a campo, tornando a técnica impraticável em avaliações veterinárias, na rotina do treinamento e durante competições esportivas. A técnica de ultra-som foi realizada facilmente e com segurança nos cavalos atletas que compuseram este experimento sem, entretanto, afetar sua programação diária, principalmente o treinamento, um elemento que é de grande importância para o cavalo em qualquer modalidade esportiva (Kearns et al., 2002a). Devido às vantagens que foram encontradas no emprego da ultra-sonografia neste experimento, sugere-se que essa técnica seja utilizada como rotina durante o treinamento e mesmo nas avaliações veterinárias durante competições de longa distância.

Pode-se constatar através da análise dos dados obtidos que, no primeiro mês de treinamento dos animais, a área de corte transversal do músculo longissimus dorsi aumentou consideravelmente e que, no segundo mês de treinamento, a espessura da camada de gordura diminuiu. Porém, há um momento em que essas medidas se estabilizaram, sendo explicado pela ausência de alteração ultra-sonográfica dessas variáveis entre 60 e 90 dias de treinamento. Verificou-se também que o treinamento aeróbico associado à suplementação prolongada com creatina, nas condições utilizadas neste experimento, levou ao aumento da medida de profundidade do músculo longissimus dorsi, causando a sua hipertrofia.

Devido a este fato, novas pesquisas devem ser desenvolvidas com o intuito de averiguar o aumento de profundidade muscular, ou até mesmo, o aumento de volume celular provocado pela ingestão prolongada de creatina em cavalos, nesta intensidade de exercício.

LAWRENCE, L. M.; JACKSON, S.; KLINE, K.; MOSER, L.; BIEL, M. Observations on body weight and condition of horses in a 150-mile endurance ride. Equine Vet Sci, v. 12, p. 320-324, 1992.

LOW, S. Y.; RENNIE, M. J.; TAYLOR, P. M. Modulation of glycogen synthesis in rat skeletal muscle by changes in cell. J Physiol, v. 495, p. 299-303, 1996.

SAMPAIO, I. B. M. (Ed.) Estatística aplicada à experimentação animal. Belo Horizonte: Fundação de Ensino e Pesquisa em Medicina Veterinária e Zootecnia, 1998.

SCHUBACK, K.; ESSÉN-GUSTAVSSON, B.; PERSSON, G. B. Effect of creatine supplementation on muscle metabolic response to a maximal treadmill exercise test in standardbred horses. Equine Vet J, v. 32, p. 533-540, 2000.

SPARLING, P. B.; CURETON, K. J. Biological determinants of the sex difference in 12-min run performance. Med Sci Sports Exerc, v. 15, p.218-223, 1983.

STROUD, M. A.; HOLLIMAN, D.; BELL, D.; GREEN, A. L.; MacDONALD, GREENHAFF, P. L.. Effect of oral creatine supplementation on respiratory gas exchange and blood lactate accumulation during steady-state incremental treadmill exercise and recovery in man. Clin Sci (London), v. 87, p. 707-710, 1994.

VANDENBERGHE, K.; GILLIS, N.; VANLEEMPUTTE, M.; VANHECKE, P.; VANSTAPEL, F.; HESPEL, P. Caffeine counteracts the ergogenic action of muscle creatine loading. J Apply Physiology, v. 80, p. 452-457, 1996. VANDENBERGHE, K.; GORIS, M.; VAN HECKE, P.; VAN LEEMPUTTE, M.; VANGERVEN, L.; HESPEL, P. Long term creatine intake is beneficial to muscle performance during resistance training. J Appl Physiol, v. 83, p. 2055-2063, 1997.

VOLEK, J. S.; KRAEMER, W. J.;BUSH, J. A.; INCLEDON, M. T.; CLACK, K. C.; LYNCH, J. M. Creatine supplementation enhances muscular performance during high-intensity resistance exercise. J Am Diet Assoc, v. 97, p. 765-770, 1997. 